\title{
TAMAÑO POBLACIONAL Y ALIMENTACIÓN DE LA NUTRIA DE RÍO (LONTRA LONGICAUDIS ANNECTENS) EN LA COSTA DE OAXACA, MEXICO
}

\author{
Ma. Antonieta Casariego-Madorell, Rurik List y Gerardo Ceballos \\ Instituto de Ecología, Universidad Nacional Autónoma de México, \\ Apartado Postal 70-275, 04510 México D. F., MÉXICO. \\ madorell@prodigy.net.mx.
}

\begin{abstract}
RESUMEN
La nutria de río neotropical (Lontra longicaudis annectens) se encuentra amenazada en México y para diseñar estrategias reales de conservación, son necesarios estudios básicos de su biología y ecología. Se evaluaron la dieta y abundancia entre julio de 1999 y agosto del 2000 en los ríos Ayuta, Copalita y Zimatán en la costa de Oaxaca. Considerando una estimación de cuatro nutrias en $147.5 \mathrm{~km}$ del río Ayuta. 86 nutrias en $330.75 \mathrm{~km}$ del río Copalita y 177 nutrias en $228.85 \mathrm{~km}$ del río Zimatán. Encontrando de forma indirecta una relación entre la abundancia de la nutria y el $\mathrm{O}_{2}$ disuelto en el agua. De 330 excretas se determinaron cuatro categorías de presa que incluyen crustáceos $(53.0 \pm 3.6 \%)$, peces $(33.1 \pm 9.9 \%)$, insectos $(9.8 \pm 7.6 \%)$ y anfibios $(4.0 \pm 3.3 \%)$. Respecto a la biomasa consumida, la presa principal fue Macrobrachium americanum, M. acanthochirus y Gobiexos mexicanus. El número más alto de peces y crustáceos se obtuvo en el río Zimatán $(\mathrm{n}=258$ muestras, 16 spp.) seguido por el río Ayuta $(\mathrm{n}=253,14 \mathrm{spp})$ y el río Copalita $(\mathrm{n}=197,16 \mathrm{spp})$. Encontrando una relación entre la frecuencia de aparición de especies de la presa en las excretas, con su abundancia $\left(\mathrm{p}<0.01 ; \mathrm{r}^{2}=0.40\right)$, así como una relación significativa entre la biomasa consumida y la abundancia de las presas. La abundancia de las presas está determinada por $\mathrm{O}_{2}$ disuelto en el agua $\left(\mathrm{p}=0.04 ; \mathrm{r}^{2}=0.09\right)$ así como la turbiedad $\left(\mathrm{p}=0.04 ; \mathrm{r}^{2}=0.22\right)$. No hubo ninguna relación entre las variables fisicoquímicas del agua con la diversidad de las presas potenciales.
\end{abstract}

Palabras clave: Lontra longicaudis, abundancia, dieta, biomasa, río, Oaxaca.

\begin{abstract}
The neotropical river otter (Lontra longicaudis annectens) is threatened in Mexico, and in order to design sound conservation strategies for its conservation, basic studies on their biology and ecology are necessary. The diet and abundance were evaluated between July, 1999 and August, 2000 in the rivers Ayuta, Copalita and Zimatán in the coast of Oaxaca. We considered estimated of four otters in $147.5 \mathrm{~km}$ the river Ayuta. 86 otters in $330.75 \mathrm{~km}$ the rive Copalita and 177 otters in $228.85 \mathrm{~km}$ the river Zimatán. We found a relation in an indirect way between the abundance of the otter and dissolved $\mathrm{O}_{2}$. From 330 scats it was determined that they use four prey categories, which include crustaceans $(53.0 \pm 3.6 \%)$, fish $(33.1 \pm 9.9 \%)$, insects $(9.8 \pm 7.6 \%)$ and amphibians $(4.0 \pm 3.3 \%)$. In relation to the biomass ingested, the main prey were Macrobrachium americanum, M. acanthochirus and Gobiexos mexicanus. The largest number of fish and crustaceans used was obtained in the river Zimatán ( $\mathrm{n}=258$ samples, $16 \mathrm{spp}$.) followed by the river Ayuta $(\mathrm{n}=253,14 \mathrm{spp})$ and the river Copalita $(\mathrm{n}=197,16 \mathrm{spp})$. We found a
\end{abstract}


relation among the frequency of appearance of prey species in the scats, with their abundance $(\mathrm{p}<0.01$; $\left.\mathrm{r}^{2}=0.40\right)$, as well as a significant relation between the consumed biomass and the abundance of the prey. Abundance of prey is determined by $\mathrm{O}_{2}$ dissolved in the water $\left(\mathrm{p}=0.04 ; \mathrm{r}^{2}=0.09\right)$ as well as turbidity $\left(p=0.04 ; r^{2}=0.22\right)$. There was no relation between the physiochemical variables of the water with the diversity of the potential prey.

Key words: Lontra longicaudis, abundance, diet, biomass, river, Oaxaca

\section{INTRODUCCIÓN}

La nutria de río Lontra longicaudis annectens es una especie de amplia distribución, sin embargo sus poblaciones han sufrido reducciones severas y ha sido extirpada de muchas localidades, por lo que se encuentra incluida en el Apéndice I de la Convención Internacional para el Tráfico de Especies de Flora y Fauna Amenazadas (CITES). En México es considerada como amenazada (SEMARNAT 2002), a consecuencia de la cacería, la destrucción de su hábitat y la contaminación de los ríos y cuerpos de agua (Alho et al. 1988; Chehébar 1991; Gallo 1986). La nutria de río se encuentra en áreas templadas y tropicales desde el noroeste de México hasta Argentina (Aranda 2000; Gallo 1996), siendo el bosque tropical caducifolio de México, el ecosistema más amenazado de los que ocupa la nutria (Ceballos \& García 1995; Janzen 1988). El estado de Oaxaca mantiene franjas continuas de selva baja caducifolia y algunos fragmentos aislados en las cercanías de los ríos, incluyendo al río Zimatán (García et al. 1992; Salas et al. 1996) que junto con el río Copalita y el río Ayuta son los ríos más importantes en el área de Huatulco. Al ser perennes y por su caudal, representan una oportunidad para realizar estudios sobre la nutria en un área en la que el conocimiento sobre esta especie es escaso, pero necesario para asegurar la permanencia de las poblaciones de nutria de río en esta región (Gallo 1996; Ruiz-Olmos et al. 2000). Por lo que en este estudio se determinó la abundancia, tamaño poblacional y los hábitos alimentarios de la nutria en los ríos Ayuta, Copalita y Zimatán en la costa de Oaxaca.

\section{MATERIAL Y MÉTODOS}

Área de estudio. Los ríos Ayuta, Copalita y Zimatán se encuentran en la costa de Oaxaca, entre los $15^{\circ} 43^{\prime}$ y $16^{\circ} 50^{\prime} \mathrm{N}$ y $95^{\circ} 45^{\prime}$ y $96^{\circ} 15^{\prime} \mathrm{W}$ y dentro de los municipios de San Pedro Huamelula, Santiago Astata y San Miguel del Puerto. El área de estudio comprende una superficie de 27,463 ha para el río Ayuta, 152,535 ha para el río Copalita y 46,081 ha para el río Zimatán (Figura 1). Estos ríos son importantes para las nutrias ya que son corrientes permanentes. También existen varias corrientes intermitentes como el río Chacalapa y lagunas costeras como Mezcalco, Garrapatero, El Rosario y La Colorada. El clima es cálido subhúmedo Aw 
(según Koeppen), con una temperatura media anual de $28^{\circ} \mathrm{C}$, una precipitación promedio anual de 700 a $800 \mathrm{~mm}$ (Rzedowski 1978). Se caracteriza por presentar una estacionalidad con dos temporadas bien definidas: la de lluvias, de mayo a octubre, y la de secas, de noviembre a abril (INEGI 1988). Los principales tipos de vegetación son el bosque tropical caducifolio o selva baja; la selva tropical subcaducifolia a la orilla de los ríos; hacia la porción norte en los sitios cercanos a las serranías se encuentran selvas tropicales subperenifolias, y en los alrededores de las lagunas costeras, franjas angostas de manglar (Rzedowski 1978; Salas-Morales 2002).

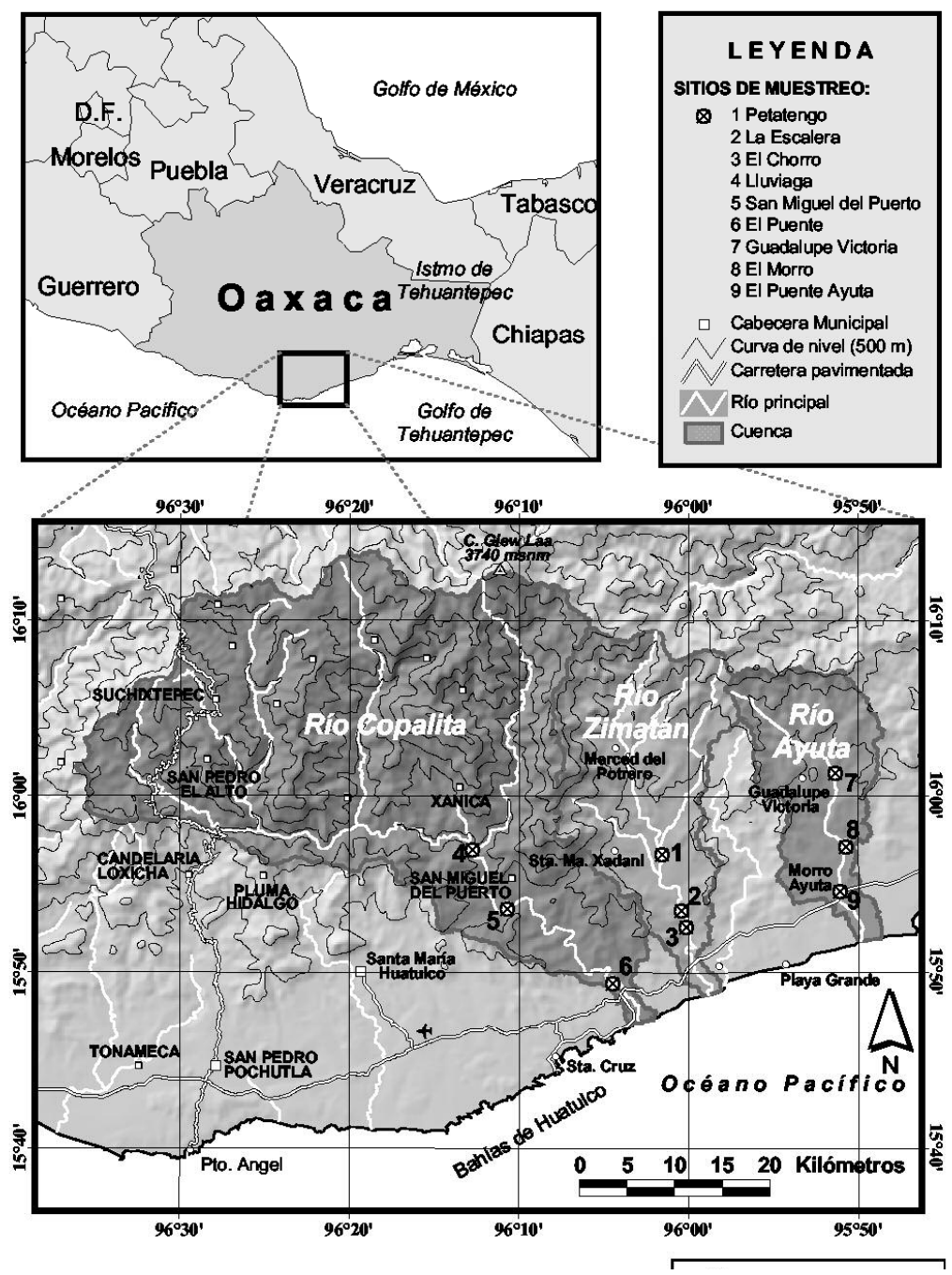

Figura 1. Ubicación geográfica del área de estudio de la nutria de río en la costa de Oaxaca. 
Caracterización del hábitat. Se realizó para cada uno de los tres ríos, se determinaron las especies de flora presentes en los alrededores y se consideraron las más abundantes para la zona. Para obtener la altura de las rocas fue por observación con puntos de referencia y cuando era posible medidas con un metro (Truper $\left.{ }^{\circledR}\right)$. El sustrato de la zona se obtuvo de acuerdo a Franco-Lopez et al (1985). Las características fisicoquímicas que se tomaron en cuenta fueron; $\mathrm{T}^{\circ}$ ambiental, $\mathrm{T}^{\circ}$ del agua, lecho (ancho real del río), cauce (área que ocupa el agua dentro del lecho), la profundidad, la velocidad del agua, la turbiedad, el $\mathrm{O}_{2}$ disuelto en el agua, alcalinidad y el $\mathrm{pH}$ (Lloyd 1992; Toweill \& Tabor 1982). Para obtener el ancho total del río, el cauce y la profundidad se utilizo un metro. La temperatura tanto ambiental como del agua se obtuvieron con un termómetro de mercurio (Brannan ${ }^{\circledR}$ ). La obtención del $\mathrm{O}_{2}$ disuelto y la alcalinidad se realizaron de acuerdo a la metodología sugerida por Franco -López et al (1985). Los reactivos y el material utilizados para llevar a cabo las pruebas fueron proporcionados por el laboratorio de botánica de la FES Iztacala. La turbiedad y la velocidad del agua fueron obtenidas de acuerdo a Contreras (1994). El pH se obtuvo con papel indicador (Merk $\left.{ }^{\circledR}\right)$. Los muestreos se realizaron durante los meses de abril a agosto del 2000. Para cada una de las pruebas se obtuvieron 135 muestras por río (Casariego-Madorell 2004).

Obtención de las muestras. Para cada uno de los tres ríos se definieron tres transectos de $2 \mathrm{~km}$ de longitud. Cada transecto incluyó una franja de $5 \mathrm{~m}$ de ancho desde la orilla hacia el interior del bosque (Ruiz-Olmos et al. 1998). El recorrido de los transectos se hizo a pie, durante un año, entre julio de 1999 y agosto del 2000, las salidas se realizaban cada mes con una duración de 7 días, realizando el conteo y colecta de los excrementos encontrados.

Tamaño poblacional. Para obtener la abundancia relativa se utilizaron dos índices;

I) El de Gallo (1996): No. de nutrias $/ \mathrm{km}=$ No. de excretas en el área/ tasa de defecación/Total de $\mathrm{km}$ recorridos.

La tasa de defecación que se utilizó es la obtenida por Gallo (1996) para dos nutrias adultas en el Zoológico Manuel Álvarez del Toro en Tuxtla Gutiérrez, Chiapas (3 excretas/día).

Sin embargo, debido a que para este índice se recomienda limpiar la zona de muestreo de excretas preexistentes y contar las excretas depositadas al día siguiente y en algunos lugares es imposible regresar a la zona de muestreo dos días seguidos tanto por la dificultad de accesar al área como por falta de equipo humano entre otros. $\mathrm{Y}$ de no seguir el índice como fue diseñado podría resultar en una sobre o subestimación de la población.

Por lo que fue necesario utilizar otro índice;

II) El de Eberhardt \& Van Etten (1956), modificado para aplicarlo a este estudio. La fórmula para convertir número de excretas por área es; $E=(N K)(E A) /(T P)$ (3) 
donde NK es el número de $\mathrm{km}$ recorridos $(2 \mathrm{~km})$, EA es el número de excretas en el área, TP el tiempo de depósito de los excrementos y 3 la tasa de defecación encontrada por Gallo (1996).

Para obtener el tamaño poblacional de la nutria en los ríos Ayuta, Copalita y Zimatán se extrapolaron los resultados obtenidos con los modelos I y II, a la longitud total aproximada de los ríos extraídas de las cartas topográficas (INEGI 1988).

Para determinar los parámetros ambientales que influyen en la presencia de la nutria para los tres ríos se realizó una regresión lineal múltiple (Myers 1986). Para comparar las abundancias relativas en los tres diferentes ríos y saber si existen o no diferencias significativas, y para comparar los índices utilizados se realizó una prueba de Kruskal - Wallis (Zar 1999).

Hábitos alimentarios. Cada excremento colectado se guardó en una bolsa de papel y se seco a temperatura ambiente (Melquist \& Hornocker 1983). Cada excremento se consideró como un evento alimentario independiente, ya que no fue posible determinar si los excrementos pertenecen al mismo animal o si son dos o más restos de una sola captura. Los excrementos se colocaron individualmente en medias de nylon y se lavaron con agua corriente, dejando secar los restos a temperatura ambiente (Emmons 1987). Una vez secos, los restos se separaron manualmente y se identificaron hasta la categoría taxonómica más baja (Helder \& de Andrade 1997). Estas determinaciones se realizaron comparando los restos obtenidos, con los peces e invertebrados acuáticos colectados en los ríos. De manera semejante se analizaron pelos, comparando la medula de las muestras con los que se obtuvieron como referencia de los mamíferos de la zona (Arita \& Aranda 1987).

Para la identificación de las especies más importantes en la dieta se utilizaron dos índices (Helder \& de Andrade 1997): 1) La frecuencia de aparición de cada especie presa en las muestras; $\mathrm{FA}=\mathrm{f}_{\mathrm{i}} / \mathrm{N}$ x 100 donde: $\mathrm{f}_{\mathrm{i}}$ es el número de excrementos en los que aparece la especie $i, \mathrm{y} \mathrm{N}$ es el número total de excrementos. 2) La proporción de aparición de cada especie presa en las muestras. $\mathrm{PA}=\mathrm{f}_{\mathrm{i}} / \mathrm{F}$ x 100 donde: $\mathrm{f}_{\mathrm{i}}$ es el número de excretas en las que aparece la especie $i$. y $\mathrm{F}$ el número total de apariciones de todas las especies en todos los excrementos, que se obtienen sumando todas las $\mathrm{f}_{\mathrm{i}}$.

Para evaluar las diferencias entre la frecuencia relativa de cada uno de los grupos de presas consumidas se aplicó la prueba de Kruskal - Wallis para los ríos Copalita y Zimatán, ya que se consideraron dos épocas de lluvia (de julio a agosto de 1999; de mayo a agosto del 2000) y una época seca (de noviembre de 1999 a abril del 2000). En el caso del río Ayuta se usó una prueba de Anova ya que sólo se consideraron dos épocas una de lluvia y una época seca (Zar 1999).

Para estimar la biomasa consumida se utilizó el método propuesto por Wise (1980), que se calcula a partir del peso seco de los restos encontrados en los excrementos y la importancia de cada presa estimada visualmente y valorada en una escala del 1 al 10. Aunque ninguno de los métodos existentes pueden estimar de 
forma precisa la importancia real de cada presa, el método de Wise (1980) para obtener la biomasa consumida fue probado controlando la dieta suministrada a nutrias en cautiverio (Jacobsen \& Hansen 1996) y actualmente se ha utilizado con $L$. longicaudis en el lago Ibera en Argentina (Gori et al. 2003). Dicho método muestra similitudes mayores con la dieta real que los métodos normalmente utilizados. No obstante este método también tiene sus limitaciones, ya que establece una relación directa entre el volumen de restos encontrados y la masa original de la presa ingerida, asumiendo que la proporción de restos producidos por los distintos tipos de presa es constante. Esto es cierto cuando comparamos por ejemplo peces con peces, pero puede ser un error cuando se comparan peces, anfibios, aves y mamíferos por las relaciones entre huesos, plumas y pelos (Pascual-Toca 2000). Es decir, en cualquier análisis de la dieta se obtienen resultados sesgados pero pueden ser utilizados con seguridad para determinar, al menos el orden relativo de consumo de las distintas presas (Carss \& Parkinson 1996).

Disponibilidad de alimento. Entre las presas potenciales para la nutria en la zona existen 22 especies de peces (Martínez - Ramírez 1999) y 7 de crustáceos (obs. pers). La técnica para cuantificar la abundancia fue la de pesca por tarraya durante 4 horas por cada río durante los meses de mayo, julio y agosto del 2000 (Montiel-Jaime 1994). Los resultados fueron expresados como número de individuos / unidad de esfuerzo (Texeira 1994).

Tanto para determinar la diversidad de las especies-presa consumidas por la nutria y la disponibilidad de las mismas se utilizó el índice de Shannon-Wiener (Sokal \& Rohlf 1981) y se aplico una prueba de Kruskal - Wallis para saber si existían diferencias significativas entre la diversidad de las presas utilizadas de los tres ríos y para cada época del año (Zar 1999).

Se realizó una regresión lineal múltiple para determinar si existe relación entre la diversidad de las especies presa y los parámetros ambientales, así como entre éstos y la abundancia de las presas. También se realizó una regresión lineal para conocer si existe relación entre la frecuencia de aparición de las especies presa en los excrementos con la abundancia de las mismas y con la biomasa consumida (Myers 1986).

\section{RESULTADOS}

Caracterización del hábitat. El río Ayuta colinda en parte con el poblado de Guadalupe Victoria y parte con el Morro Ayuta. Entre las especies más abundantes de flora a la orilla del río se encuentran miembros de la familia Bignoniaceae como Astianthus viminalis y de la familia Leguminosae como Andira inermis y Albizia occidentalis. Existen rocas de caliza con paredes de aproximadamente $3 \mathrm{~m}$ hasta zonas con arena. En esta área no se tiene la posibilidad de encontrar sitios ideales 
para madrigueras como lo mencionan Gallo (1989) y Pardini \& Trajano (1999). Durante la época seca varias partes del río se secaron al menos durante el año del muestreo. Las características fisicoquímicas de acuerdo a la metodología de Contreras (1994) y Franco-López et al (1985) indican un río poco profundo en su mayoría, con corrientes moderadas en ambas épocas del año. Durante la época de lluvia, el agua se enturbia en menos del $20 \%$ y mantiene una concentración media de $\mathrm{O}$ disuelto en el agua de $3.4 \pm 0.1 \mathrm{mg} / \mathrm{l} \mathrm{y} \mathrm{pH} \mathrm{de} 6-7$. La temperatura del ambiente $\mathrm{se}^{2}$ mantiene con un promedio de $29.6^{\circ} \mathrm{C}$ y $25.7^{\circ} \mathrm{C}$ la temperatura del agua. En este río se ha observado la cacería y pesca de subsistencia principalmente, a la orilla del río se pueden observar sembradíos de papaya.

El río Copalita abarca tres diferentes poblados; Yuviaga en la parte alta, en el centro el poblado de San Miguel del Puerto y en la parte baja Copalita. Este río es el más largo de la zona; su cuenca ha sido alterada por lo que durante la época de lluvia, el agua que lleva es de color café intenso lo que no se observa en los otros dos ríos. Encontrando similitud en las especies de flora más abundantes con el río Ayuta, se encontraron también ejemplares de Pithecellobium dulce, Mazuma ulmifolia, Tabebuia sp, y Ceiba pentandra. No es común encontrar rocas mayores de $2 \mathrm{~m}$. Aunque se tiene una pared de aproximadamente $5 \mathrm{~m}$, donde se encontraron grietas en las rocas, área donde se registraron excretas sólo en la época seca; en lluvias se torna imposible caminar por la orilla de las paredes. A principios de la época de lluvias se encontraron huellas en las zonas de arena y excretas sobre los troncos caídos a causa del huracán "Paulina".

De acuerdo a las características fisicoquímicas se observa que es un río con zonas profundas y corrientes rápidas, puede llegar a tener más del $50 \%$ de turbiedad durante la época de lluvias, manteniendo una concentración media de $\mathrm{O}$ disuelto en el agua de $3.4 \pm 0.2 \mathrm{mg} / \mathrm{l}$ aunque no se altera el $\mathrm{pH}$ que es de $6-7$, con una temperatura promedio en el ambiente de $29^{\circ} \mathrm{C}$ y una temperatura de $24.4^{\circ} \mathrm{C}$ en el agua. En este río se práctica el recorrido en kayak, ecoturismo y la pesca de subsistencia. Principalmente se tienen sembradíos de café en las partes altas del río y de papaya y toronja a la orilla de algunos tramos del río, cerca de la bocana en el transecto 1 (Puente Copalita) se extrae material para construcción constituyendo un área altamente perturbada.

El río Zimatán pertenece a la cuenca del Zimatán, con un gran número de brazos que se extienden en toda el área. Encontrando que las especies más abundantes de flora son las mismas que se encontraron en el río Ayuta y en el Copalita, aunque también se encontraron cactáceas de los géneros Mammillaria, Neobuxbaumia y Stenocereus, así como diversas especies del género Acacia y Mimosa. En esta zona se encuentran peñascos de hasta $5 \mathrm{~m}$ de altura donde se observa escasa vegetación, en su mayoría cactáceas. Se tienen también áreas de arena con extensa cobertura vegetal en las cuales se han encontrado huellas y excretas de la nutria. De acuerdo a 
las características fisicoquímicas muestran un río en su mayoría profundo con corrientes rápidas y pozas de profundidad media, la turbiedad nunca fue de más de $20 \%$ y el O disuelto en el agua se encuentra dentro de los parámetros normales de $6.1 \pm 0.3 \mathrm{mg} / 1$ y un $\mathrm{pH}$ de 7 requeridos para poblaciones viable de peces (Lloyd 1992). Con una temperatura promedio en el ambiente de $30.2^{\circ} \mathrm{C}$ y una temperatura de $26.2^{\circ} \mathrm{C}$ en el agua. En este río se ha observado el ecoturismo sobre todo en la parte baja llamado "el Chorro" encontrando también ganado, aunque la mayoría de los habitantes practican la cacería de subsistencia y en menor proporción para venta.

Abundancia relativa. Se encontraron 4 excretas en el río Ayuta, 82 excretas en el río Copalita y 208 excretas en el río Zimatán. El mayor número de excretas encontradas fue en los meses de octubre a marzo, finalizando la temporada de lluvia y abarcando la época seca. La abundancia y el tamaño poblacional obtenidos con los dos modelos utilizados fueron muy variados. Teniendo $0.056 \pm 0.036$ nutrias $/ \mathrm{km}$ con el modelo I y $0.005 \pm 0.0026$ nutrias $/ \mathrm{km}$ con el modelo II para el río Ayuta. $0.413 \pm$ 0.106 nutrias $/ \mathrm{km}$ con el modelo I y $0.111 \pm 0.042$ nutrias $/ \mathrm{km}$ con el modelo II para el río Copalita. $1.095 \pm 0.220$ nutrias $/ \mathrm{km}$ con el modelo I y $1.095 \pm 0.220$ nutrias $/ \mathrm{km}$ con el modelo I y $0.157 \pm 0.044$ nutrias $/ \mathrm{km}$ con el modelo II para el río Zimatán.

Se obtuvieron diferencias significativas en las abundancias de los ríos, para el modelo I $(\mathrm{H}=24.05, \mathrm{gl}=2, \mathrm{P}<0.05)$ entre el río Zimatán y el río Ayuta $(\mathrm{Q}=4.78, \mathrm{P}$ $<0.05)$ y entre los ríos Copalita y Ayuta $(\mathrm{Q}=2.91, \mathrm{P}<0.05)$. No hubo diferencias entre el río Copalita y Zimatán $(\mathrm{Q}=2.05, \mathrm{P}>0.05)$. Con el modelo II también se encontraron diferencias $(\mathrm{H}=21.51, \mathrm{gl}=2, \mathrm{P}<0.05)$ entre el río Zimatán y el Ayuta $(\mathrm{Q}=4.34, \mathrm{P}<0.05)$, así como entre el río Ayuta y el Copalita $(\mathrm{Q}=3.33, \mathrm{P}<0.05)$. No hubo diferencias entre los ríos Copalita y Zimatán $(\mathrm{Q}=1.22, \mathrm{P}>0.05)$.

Para el río Ayuta no se encontraron diferencias entre los dos modelos $(\mathrm{H}=0.21, \mathrm{gl}=1$, $\mathrm{P}>0.05)$. Para el río Copalita se encontraron diferencias significativas $(\mathrm{H}=6.28, \mathrm{gl}=1, \mathrm{P}<0.05)$ entre ambos modelos $(\mathrm{Q}=2.50, \mathrm{P}<0.05)$ al igual que para el río Zimatán $(\mathrm{H}=13.28, \mathrm{gl}=1, \mathrm{P}<0.05)$ entre el modelo I y II $(\mathrm{Q}=3.64, \mathrm{P}<0.05)$.

Tamaño poblacional. Considerando un promedio entre las estimaciones obtenidas con ambos modelos (Cuadro 1) y extrapolado a las longitudes de los ríos se obtiene un tamaño poblacional aproximado de 4 nutrias en $147.5 \mathrm{~km}$ del río Ayuta. 86 nutrias en $330.75 \mathrm{~km}$ del río Copalita y 177 nutrias en $228.85 \mathrm{~km}$ del río Zimatán. El análisis de regresión múltiple indica que existe una relación significativa entre la abundancia de la nutria con el $\mathrm{O}_{2}$ disuelto en el agua aunque con bajo valor predictivo $(\mathrm{p}<0.001$; $\mathrm{r}^{2}=0.23$ ) (Figura 2). Para las demás variables no se encontró relación significativa.

Hábitos alimentarios. Se analizaron 281 excrementos para los tres ríos, 9 para el río Ayuta, 99 para el río Copalita y 173 para el río Zimatán. La alimentación de la nutria de río en esta zona se compone principalmente de crustáceos $(53.0 \pm 3.7 \%)$, peces $(33.1 \pm 9.9 \%)$, insectos $(9.8 \pm 7.6 \%)$ y anfibios $(4.0 \pm 3.3 \%)$, Macrobrachium americanum $(32.0 \pm 8.8 \%)$ y el pez Gobiexos mexicanus $(14.7 \pm 2.1 \%)$ destacan 
Cuadro 1. Abundancia relativa de L. longicaudis para los ríos Ayuta, Copalita y Zimatán obtenidas con el modelo (I) de Gallo (1996) y el modelo (II) Eberhardt - Van Etten (1956). (Los datos se representan como el promedio \pm error estándar). Así como la estimación del tamaño poblacional de L. longicaudis para los ríos Ayuta, Copalita, y Zimatán (Los datos se representan como promedio \pm error estándar).

Modelos Abundancia relativa (nutria $/ \mathrm{km}$ )

Río Ayuta Río Copalita Río Zimatán

I $\quad 0.056 \pm 0.036$

$0.413 \pm 0.106$

$1.095 \pm 0.220$

$8.194 \pm 5.36$

Tamaño poblacional

\begin{tabular}{|c|c|c|c|c|c|c|}
\hline & Río Ayuta & Río Copalita & Río Zimatán & $\begin{array}{l}\text { Río Ayuta } \\
\text { (147.5 km) }\end{array}$ & $\begin{array}{c}\text { Río Copalita } \\
(330.75 \text { km) }\end{array}$ & $\begin{array}{l}\text { Río Zim atán } \\
(228.85 \text { km) }\end{array}$ \\
\hline I & $0.056 \pm 0.036$ & $0.413 \pm 0.106$ & $1.095 \pm 0.220$ & $8.194 \pm 5.36$ & $136.50 \pm 35.18$ & $309.99 \pm 62.21$ \\
\hline & $0.005 \pm 0.0026$ & $0.111 \pm 0.042$ & $0.157 \pm 0.044$ & $0.777 \pm 0.38$ & $36.76 \pm 14.06$ & $44.38 \pm 12.54$ \\
\hline
\end{tabular}

como presas principales. Se encontraron diferencias significativas en el consumo de las presas $(\mathrm{H}=40.60, \mathrm{gl}=3, \mathrm{p}<0.05)$. Las diferencias se encontraron entre los grupos de crustáceos y anfibios $(\mathrm{Q}=5.28, \mathrm{p}<0.05)$, crustáceos e insectos $(\mathrm{Q}=3.79, \mathrm{p}<0.05)$ así como entre peces y anfibios $(\mathrm{Q}=4.64, \mathrm{p}<0.05)$ y peces e insectos $(\mathrm{Q}=3.13$, $\mathrm{p}<$ 0.05). No se encontraron diferencias significativas entre el consumo de crustáceos y peces $(\mathrm{Q}=0.74, \mathrm{p}>0.05)$ e insectos y anfibios $(\mathrm{Q}=1.48, \mathrm{p}>0.05)$. También se encontraron diferencias significativas en el consumo de las presas entre los tres ríos $(\mathrm{H}=10.64, \mathrm{gl}=3, \mathrm{p}<0.05)$. Las diferencias se encontraron entre el río Ayuta y Copalita $(\mathrm{Q}=2.75, \mathrm{p}<0.05)$ y entre el río Ayuta y Zimatán $(\mathrm{Q}=2.99, \mathrm{p}<0.05)$, sin encontrar diferencias entre los ríos Copalita y Zimatán $(\mathrm{Q}=0.32, \mathrm{p}>0.05)$.

Los excrementos de las nutrias en el río Ayuta incluyeron $50.0 \%$ de crustáceos, $14.3 \%$ de peces, $10.7 \%$ de anfibios y $25.0 \%$ de insectos. Para la temporada de lluvias se encontró una proporción mayor de crustáceos $(57.1 \%)$, peces $(21.4 \%)$ y en menor proporción los anfibios (14.3\%) e insectos (7.1\%), destacando a M. acanthochirus $(21.4 \%)$ con una mayor proporción de aparición. Para la época de secas como presas principales se encontraron a los crustáceos $(42.9 \%)$ e insectos $(42.9 \%)$ y en menor proporción a los peces $(7.1 \%)$ y anfibios $(7.1 \%)$. Donde las principales presas fueron M. americanum (21.4\%), así como ortópteros y alacranes de la familia Butidae. No se encontraron diferencias significativas entre la temporada de lluvias y la temporada seca (ANOVA $\mathrm{F}=0.052, \mathrm{p}=0.800$ ) como tampoco se encontraron diferencias entre el consumo de las diferentes categorías $(\mathrm{H}=1.46, \mathrm{gl}=3, \mathrm{p}>0.05)$. 


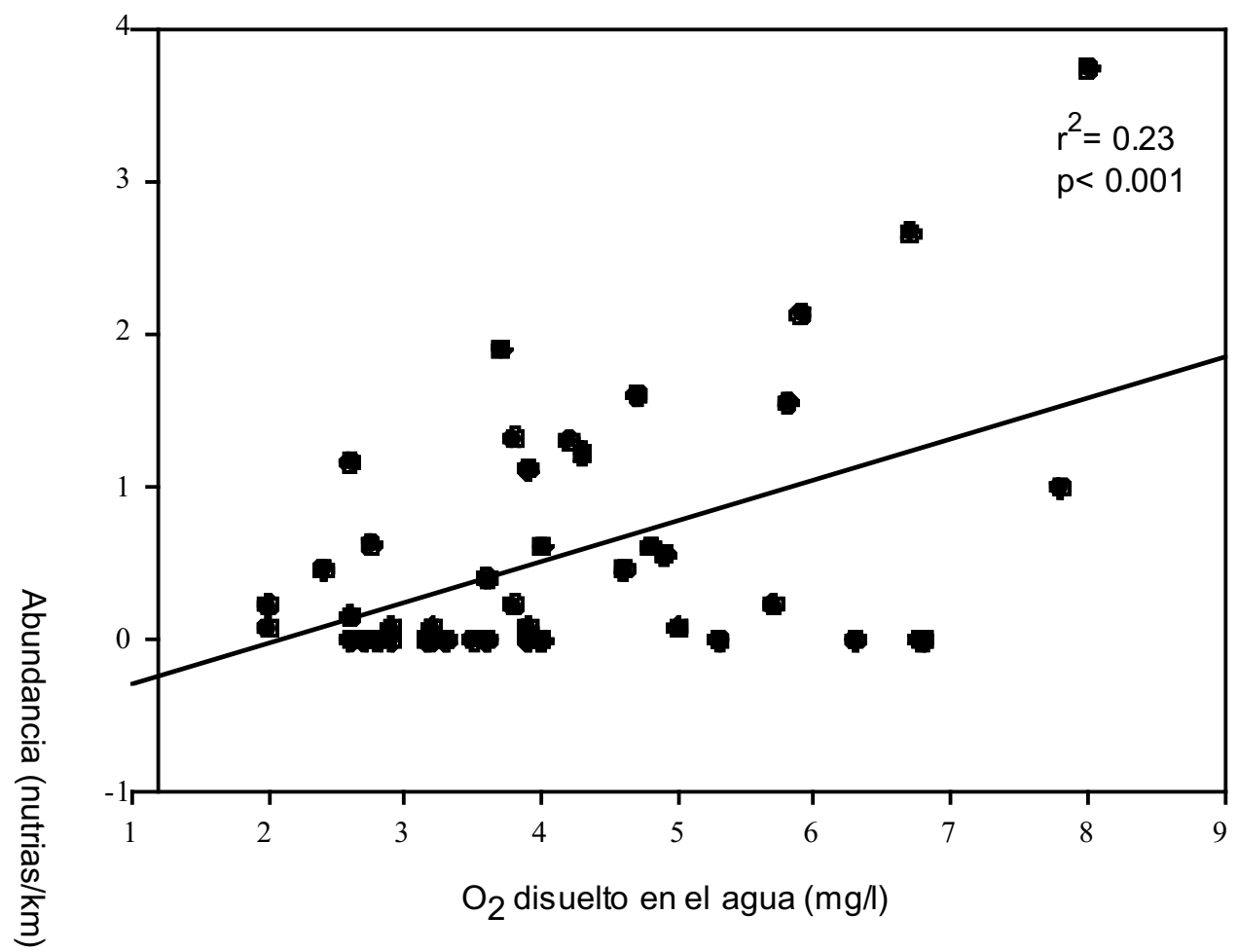

Figura 2. Relación entre la abundancia de la nutria y el $\mathrm{O}_{2}$ disuelto en el agua.

Para el río Copalita se encontraron en total el $48.8 \%$ de crustáceos, el $47.9 \%$ de peces, el $0.4 \%$ de anfibios y el $2.9 \%$ de insectos. En este río la mayor presa de la nutria durante la época de secas fueron los crustáceos $(54.5 \%)$, seguidos por los peces $(41.5 \%)$, insectos $(3.4 \%)$ y anfibios $(0.6 \%)$, las principales presas fueron $M$. americanum y $G$. mexicanus. La época de lluvia se divide en dos partes, en las primera se encontraron crustáceos $(30.2 \%)$ y peces $(69.8 \%)$, en la segunda fueron crustáceos (39.1\%), peces (56.5\%) e insectos (4.3\%), destacando M. americanum, Atya margaritacea, G. mexicanus y especies de la familia Eleotridae. No se encontraron diferencias significativas entre el consumo de presas durante la temporada de secas y las dos de lluvias $(\mathrm{H}=2.65, \mathrm{gl}=2, \mathrm{p}>0.05)$, ni entre las categorías de las presas consumidas $(\mathrm{H}=4.44, \mathrm{gl}=3, \mathrm{p}>0.05)$.

Para el río Zimatán se encontraron el $60.3 \%$ de crustáceos, el $37.1 \%$ de peces, el $1.0 \%$ de anfibios y el $1.6 \%$ de insectos. En este río la mayor presa de la nutria durante la época de secas fueron los crustáceos $(62.8 \%)$, seguidos por los peces $(34.5 \%)$ y al final los insectos $(2.2 \%)$ y anfibios $(0.4 \%)$, las principales presas 
fueron M. americanum y G. mexicanus. La época de lluvia se divide en dos partes, en las primera se encontraron crustáceos $(61.5 \%)$, peces $(33.3 \%)$ y anfibios $(5.1 \%)$, en la segunda fueron crustáceos (47.7\%) y peces $(52.3 \%)$, destacando $M$. americanum, G. mexicanus y especies de la familia Eleotridae. Hubo diferencias significativas en el consumo de presas durante las tres temporadas del año $(\mathrm{H}=$ 18.96, $\mathrm{gl}=2, \mathrm{p}<0.05)$. Las diferencias se encontraron entre la época seca y la primera de lluvias $(\mathrm{Q}=2.59, \mathrm{p}<0.05)$, y con la segunda temporada de lluvias $(\mathrm{Q}=$ $4.09, \mathrm{p}<0.05)$. No hubo diferencias entre ambas temporadas de lluvias $(\mathrm{Q}=1.50$, $\mathrm{p}>0.05)$. No se encontraron diferencias entre las categorías de presas consumidas $(\mathrm{H}=1.83, \mathrm{gl}=3, \mathrm{p}>0.05)$.

Las presas principales en la alimentación de la nutria estimada a través de la biomasa consumida para los tres ríos durante todo el año fueron para el río Ayuta un consumo del $36.3 \%$ de M. americanum y $35.5 \%$ de M. acanthochirus. Para el río Copalita un consumo del $38.8 \%$ de M. americanum y $21.5 \%$ de G. mexicanus y para el río Zimatán se obtuvo un consumo de $54.8 \%$ de M. americanum y 20.6 de $G$. mexicanus. El consumo de las demás presas fue menos del $10 \%$ para los tres ríos (Cuadro 2).

La diversidad total obtenida de cada uno de los ríos y de las diferentes estaciones se tiene que la mayor diversidad trófica se presentó en el río Copalita $(\mathrm{H}=1.048$, $\mathrm{Hmax}=4.523, \mathrm{E}=0.231)$ seguida del río Zimatán $\left(\mathrm{H}^{\prime}=0.849, \mathrm{Hmax}=4.247, \mathrm{E}=\right.$ $0.199)$ y el río Ayuta $\left(H^{\prime}=1.038, \operatorname{Hmax}=3.70, E=0.280\right)$. Se encontraron diferencias significativas $(\mathrm{H}=9.63, \mathrm{gl}=2 \mathrm{p}<0.05)$, entre la diversidad del río Ayuta y los ríos Copalita $(\mathrm{Q}=2.74, \mathrm{p}<0.05)$ y Zimatán $(\mathrm{Q}=2.82, \mathrm{p}<0.05)$. No se encontraron diferencias significativas entre el río Copalita y Zimatán $(\mathrm{Q}=0.20, \mathrm{p}>0.05)$. Tampoco se encontraron diferencias significativas entre la época de lluvia y la época de seca para el río Ayuta $(\mathrm{t}=57.00, \mathrm{gl}=7, \mathrm{p}>0.05)$. Para el río Copalita se encontraron diferencias significativas $(H=13.08, g l=2, p<0.05)$, entre la temporada seca y la primera $(\mathrm{Q}=2.75, \mathrm{p}<0.05)$ y segunda temporadas de lluvia $(\mathrm{Q}=3.11, \mathrm{p}<$ $0.05)$. No existieron diferencias significativas entre ambas temporadas de lluvia $(\mathrm{Q}=$ $0.68, \mathrm{p}>0.05)$. En el río Zimatán se encontraron diferencias significativas $(\mathrm{H}=9.62$, $\mathrm{gl}=2, \mathrm{p}<0.05)$, entre la temporada seca y la primera $(\mathrm{Q}=2.56, \mathrm{p}<0.05)$ y segunda temporada de lluvia $(\mathrm{Q}=2.40, \mathrm{p}<0.05)$. No hubo diferencias significativas entre ambas temporadas de lluvia $(\mathrm{Q}=0.36, \mathrm{p}>0.05)$.

Disponibilidad de alimento. En el muestreo que se realizó en los ríos se obtuvieron únicamente 12 especies de peces, considerando también especies de la familia Eleotridae las cuales no pudieron ser determinadas en los excrementos y 7 especies de crustáceos. El mayor número de presas tanto de peces como de crustáceos fueron obtenidas en el río Zimatán (258 individuos y 16 especies) seguido del río Ayuta (253 individuos y 14 especies) y el Copalita (197 individuos y 16 especies) (Cuadro 3). 
Casariego-Madorell, List \& Ceballos: Tamaño poblacional y alimentación de la nutria de río

Cuadro 2. Biomasa consumida B. c. \% (Peso seco del excremento x la evaluación visual de los restos encontrados de cada especie con un valor del 1 al 10) en orden de importancia para los ríos Ayuta, Copalita y Zimatán durante las diferentes épocas del año entre los meses de julio de 1999 a agosto del 2000.

\begin{tabular}{|c|c|c|c|c|c|c|c|c|c|c|}
\hline \multirow{3}{*}{ Especies-presa } & \multicolumn{3}{|c|}{ Ayuta } & \multicolumn{4}{|c|}{ Copalita } & \multicolumn{3}{|c|}{ Zimatán } \\
\hline & Total & Lluvias & Secas & Total & Lluvias & Secas & Luvias & Total & Lluvias & Secas \\
\hline & \multicolumn{3}{|c|}{$\%$ de biomasa consumida } & \multicolumn{4}{|c|}{$\%$ de bioma sa consumida } & \multicolumn{3}{|c|}{$\%$ de biomasa const } \\
\hline \multicolumn{11}{|l|}{ CRUSTACEOS } \\
\hline $\begin{array}{l}\text { Macrobrachium } \\
\text { americanum }\end{array}$ & 33.3 & 16.3 & 40.5 & 38.8 & 21.8 & 41.6 & 32.9 & 54.8 & 41.2 & 55. \\
\hline M. acanthochirus & 35.5 & 43.9 & / & 3.1 & 1.9 & 3.6 & / & / & 6.1 & 0.5 \\
\hline M. hobbsi & 6.0 & 7.5 & / & 0.5 & / & 0.4 & 2.4 & 0.5 & 0.8 & $0 . \varepsilon$ \\
\hline M. occidentales & 5.5 & / & 10.7 & 2.0 & / & 2.5 & / & 1.2 & 0.9 & 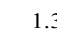 \\
\hline M. olfersii & 2.6 & / & 5.0 & / & / & 0.3 & / & 0.2 & / & 0.2 \\
\hline Atya margaritacea & 6.4 & 5.8 & / & 4.7 & / & 5.3 & 5.2 & 5.7 & 6.0 & 5.5 \\
\hline A. sp. & / & / & I & l & 0.2 & 1.2 & / & 0.5 & / & 0.6 \\
\hline \multicolumn{11}{|l|}{ PECES } \\
\hline $\begin{array}{l}\text { Gobiexos } \\
\text { mexicanus }\end{array}$ & 8.8 & 6.3 & 7.1 & 25.5 & 10.6 & 22.4 & 27.9 & 20.6 & 24.4 & 22. \\
\hline $\begin{array}{l}\text { Pomadasys } \\
\text { bayanus }\end{array}$ & / & / & / & 2.8 & 5.6 & 2.2 & 4.6 & 3.0 & 1.0 & $1 . \varepsilon$ \\
\hline Eleotridae & / & / & / & 8.6 & 19.1 & 7.8 & 2.5 & 2.1 & / & $1 . \varepsilon$ \\
\hline $\begin{array}{l}\text { Gobiomorus } \\
\text { maculatus }\end{array}$ & 7.6 & 9.4 & / & I & / & / & / & 1.5 & / & 1.t \\
\hline $\begin{array}{l}\text { Agonostomus } \\
\text { monticola }\end{array}$ & / & / & / & 6.2 & 17.3 & 4.9 & 4.6 & 1.4 & 2.7 & $1 . z^{2} \quad-1$ \\
\hline Poecilia sphenops & / & I & / & 1.0 & 3.0 & 0.9 & I & 0.3 & 0.4 & 0.2 \\
\hline $\begin{array}{l}\text { Awaous } \\
\text { transandeanus }\end{array}$ & / & / & / & 2.4 & 4.8 & 0.7 & 15.4 & 0.4 & / & $0 . \varepsilon$ \\
\hline $\begin{array}{l}\text { Sicydium } \\
\text { punctatum }\end{array}$ & I & / & / & 1.3 & 1.9 & 1.3 & I & 0.1 & / & 0.1 \\
\hline $\begin{array}{l}\text { Eucinostumos } \\
\text { currani }\end{array}$ & / & / & l & 0.9 & 5.3 & 0.3 & / & I & & \\
\hline Lutjanus aratus & / & / & / & 0.5 & / & 0.6 & / & 0.4 & 3.5 & 0.1 \\
\hline Pez no identificado & / & / & ' & 1.4 & 5.4 & 1.0 & ' & 3.6 & 4.6 & 4.2 \\
\hline \multicolumn{11}{|l|}{ ANF IBIOS } \\
\hline No identificado & 8.5 & 8.3 & 3.6 & 0.1 & / & 0.2 & / & 0.9 & 1.6 & 0.1 \\
\hline \multicolumn{11}{|l|}{ INSECTOS } \\
\hline Corydalidae & / & / & / & / & / & I & / & 0.5 & / & $0 . t$ \\
\hline Orthóptera & 4.1 & / & 8.0 & / & / & / & / & 0.2 & / & 0.2 \\
\hline Coleop tera & 0.8 & / & 1.7 & 0.8 & / & 1.0 & / & / & / & / \\
\hline Himenóptera & 2.3 & 2.3 & 0.8 & 0.4 & / & 0.1 & 4.7 & / & / & / \\
\hline Buthidae & 9.9 & / & / & 0.2 & / & 0.2 & / & / & / & / \\
\hline
\end{tabular}


Cuadro 3. Número de peces y crustáceos obtenidos durante mayo, julio y agosto del 2000 en los ríos de Ayuta, Copalita y Zimatán, representados como organismos/4 hrs hombre para cada muestreo.

\begin{tabular}{lccccccccc}
\hline & \multicolumn{1}{c}{ AYUTA } & \multicolumn{2}{c}{ COPALITA } & \multicolumn{3}{c}{ ZIMATÁN } \\
\hline & May & Jul & Ago & May & Jul & Ago & May & Jul & Ago \\
\hline ESPECIES-PRESA & & & & & & & & & \\
\hline
\end{tabular}

\section{CRUSTACEOS.}

Palaemonidae

$\begin{array}{lccccccccc}\text { M. americanum } & 6 & 2 & 7 & 7 & 4 & 11 & 14 & 9 & 5 \\ \text { M. acanthochirus } & 1 & 2 & 0 & 1 & 2 & 1 & 1 & 2 & 0 \\ \text { M. hobbsi } & 2 & 0 & 0 & 2 & 0 & 0 & 3 & 4 & 14 \\ \text { M. occidentalis } & 1 & 0 & 0 & 1 & 0 & 1 & 1 & 0 & 0 \\ \text { M. olfersii } & 0 & 2 & 2 & 4 & 0 & 0 & 21 & 2 & 0\end{array}$

Atyidae

Atya margaritacea

Atya sp.

$\begin{array}{lllll}2 & 2 & 0 & 5 & 2 \\ 0 & 0 & 0 & 0 & 0\end{array}$

$\begin{array}{lccc}1 & 16 & 2 & 0 \\ 0 & 2 & 0 & 1\end{array}$

\section{PECES}

Gobiesocidae

$\begin{array}{llllllllll}\text { Gobiexos mexicanus } & 2 & 2 & 2 & 9 & 10 & 12 & 10 & 14 & 0\end{array}$

Eleotridae

$\begin{array}{llllllllll}\text { Eleotris picta } & 2 & 4 & 0 & 4 & 0 & 1 & 3 & 0 & 0 \\ \text { Gobiomorus maculatus } & 0 & 0 & 0 & 0 & 2 & 0 & 2 & 0 & 0 \\ \text { Gobiomorus polylepis } & 6 & 0 & 3 & 20 & 14 & 17 & 7 & 24 & 0\end{array}$

Mugilidae

$\begin{array}{llllllllll}\text { Agonostomus monticola } & 6 & 0 & 13 & 28 & 4 & 6 & 13 & 14 & 0\end{array}$

Haemulidae

$\begin{array}{llllllllll}\text { Pomadasys bayanus } & 6 & 4 & 0 & 4 & 0 & 2 & 4 & 6 & 0\end{array}$

Gobiidae

$\begin{array}{llllllllll}\text { Awaous transandeanus } & 2 & 0 & 2 & 12 & 2 & 0 & 2 & 0 & 0 \\ \text { Sicydium punctatum } & 0 & 4 & 14 & 4 & 0 & 0 & 30 & 2 & 28\end{array}$

Poecilidae

$\begin{array}{llllllllll}\text { Poecilia sphenops } & 152 & 0 & 0 & 0 & 0 & 0 & 0 & 0 & 0\end{array}$

Gerridae

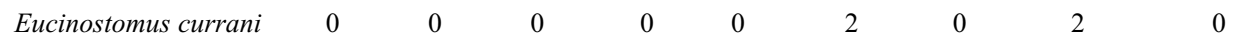

Lutjanidae

Lutjanus aratus

$0 \quad 0$

$\begin{array}{lll}0 & 0 & 0\end{array}$

20

$0 \quad 0$

\section{Total}

$188 \quad 22$

$43 \quad 101 \quad 40$

$56 \quad 129$

81

48 
La mayor diversidad trófica de las presas se presentó en el río Zimatán $\left(\mathrm{H}^{\prime}=1.00\right.$, $\mathrm{Hmax}=4.00, \mathrm{E}=0.250)$ seguida del río Copalita $\left(\mathrm{H}^{\prime}=0.95, \mathrm{Hmax}=4.00, \mathrm{E}=0.23\right)$ y el río Ayuta $\left(\mathrm{H}^{\prime}=0.69, \mathrm{Hmax}=3.80, \mathrm{E}=0.18\right)$. No hubo diferencias significativas entre la diversidad de los tres ríos $(\mathrm{H}=0.11, \mathrm{gl}=2, \mathrm{p}>0.05)$. Se encontraron diferencias significativas entre la diversidad de las presas potenciales para el río Ayuta $(\mathrm{H}=18.67$, $\mathrm{gl}=2, \mathrm{p}<0.05)$, entre julio y mayo $(\mathrm{Q}=3.89, \mathrm{p}>0.050)$, y entre mayo y agosto $(\mathrm{Q}=3.13$, $\mathrm{p}<0.05)$. Entre el mes de julio y agosto no se encontraron diferencias $(\mathrm{Q}=0.56, \mathrm{p}<$ 0.05). En el río Copalita no se encontraron diferencias significativas $(\mathrm{H}=3.79, \mathrm{gl}=2.00$, $\mathrm{p}>0.05)$ ni en el río Zimatán $(\mathrm{H}=3.09, \mathrm{gl}=2, \mathrm{p}>0.05)$.

$\mathrm{El}$ análisis de regresión múltiple indica que existe una relación significativa entre la abundancia de las presas con el $\mathrm{O}_{2}$ disuelto en el agua al igual que con la turbidez, aunque con bajo valor predictivo para ambas (Figura 3). Para las demás variables no se encontró relación significativa.

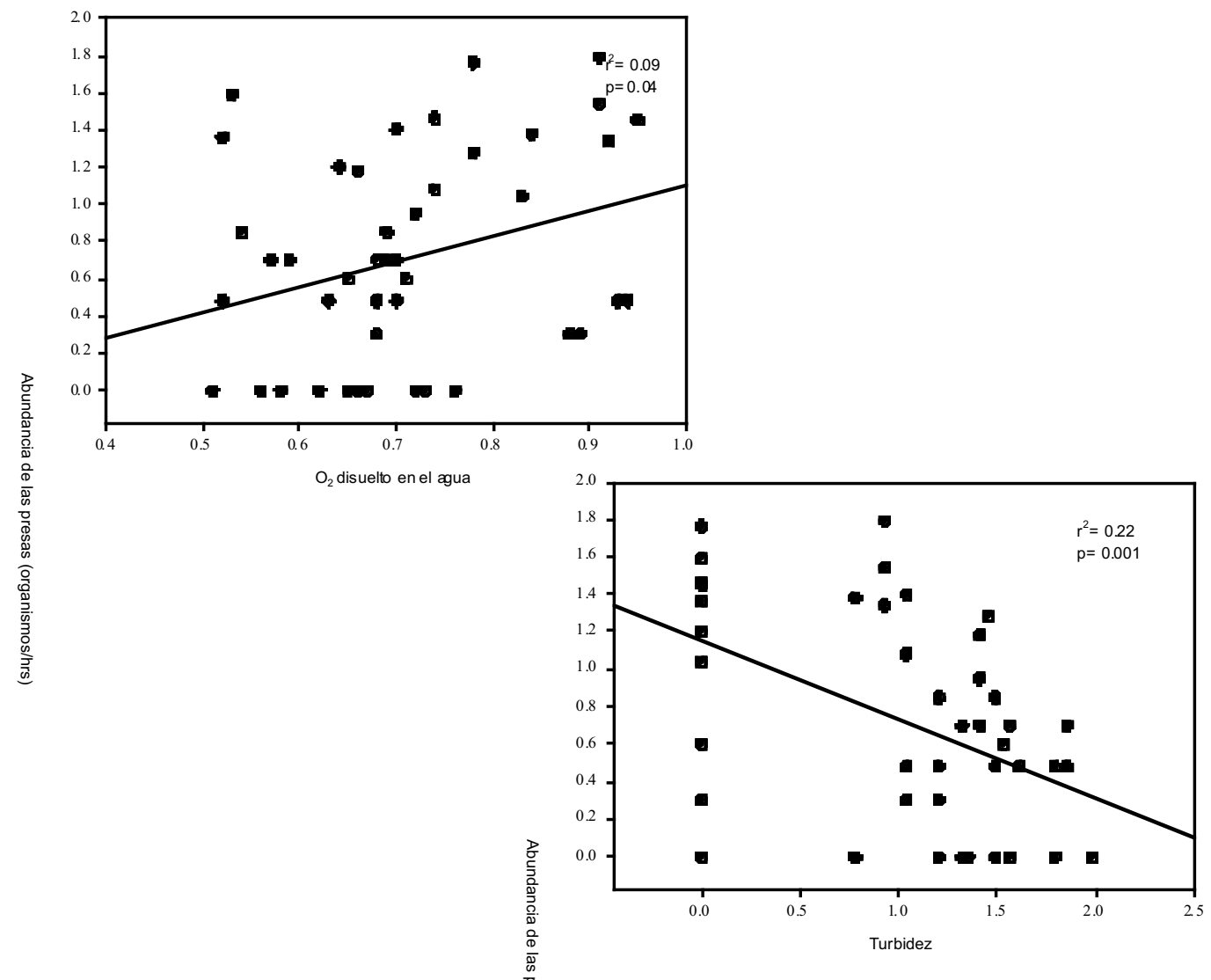

Figura 3. a) Relación entre la abundanciağ de las presas y el $\mathrm{O}_{2}$ disuelto en el agua $\left(\mathrm{r}^{2}=0.09 ; \mathrm{p}=0.04\right)$. b) Relación entre la abundancia de las pre 
Se encontró relación entre la frecuencia de aparición de las especies - presa en los excrementos de los meses de mayo, julio y agosto con la abundancia de éstas, así como con la biomasa consumida aunque de bajo valor predictivo (Figura 4).

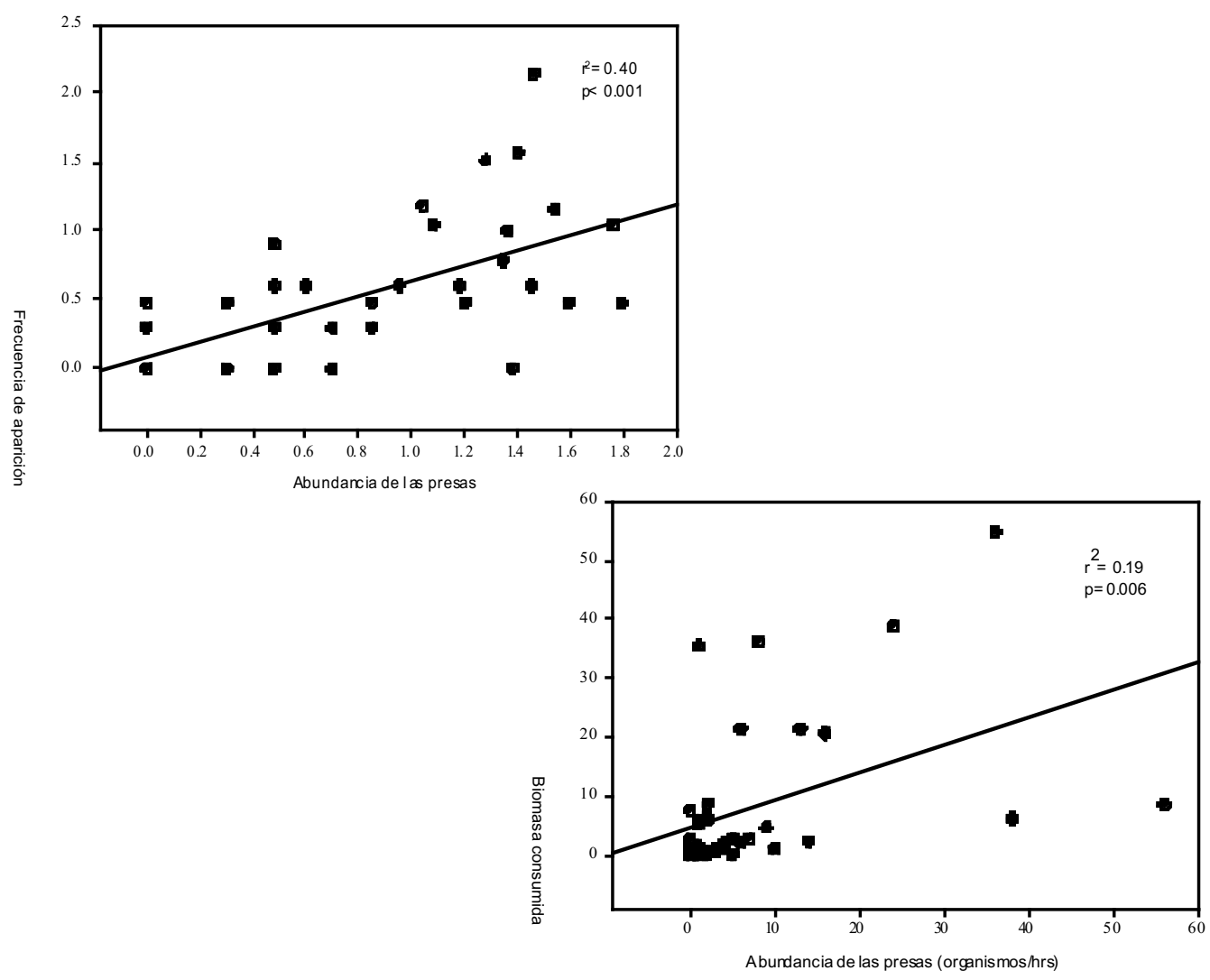

Figura 4. a) Relación entre la frecuencia relativa de especies presa en los excrementos y la abundancia de las presas en los ríos Ayuta, Copalita y Zimatán $\left(\mathrm{r}^{2}=0.40 ; \mathrm{p}=0.001\right)$. b) Relación entre la biomasa consumida de las especies presa y la abundancia de éstas $\left(\mathrm{r}^{2}=0.19 ; \mathrm{p}=0.006\right)$.

\section{DISCUSIÓN}

Abundancia relativa y tamaño poblacional. La abundancia y el tamaño poblacional obtenidos con los dos modelos fueron bajos para el río Ayuta, esto se debió al reducido número de excrementos encontrados en el río, lo cual puede reflejar un menor uso de este río o bien a que durante la sequía el nivel del agua baja 
considerablemente ocasionando que uno del los transectos no fue completado debido a que no se obtuvo el permiso de las autoridades, por lo que se considero como si no se hubieran encontrado rastros. Como se esperaba, el mayor número de rastros y la mayor abundancia de nutrias se encontraron en el río Zimatán, que es el que presenta las mejores condiciones, como grandes bloques rocosos y lugares apropiados para poder construir madrigueras (Gallo 1989; Pardini \& Trajano 1999) de hecho se lograron observar algunas madrigueras.

Se encontró relación entre la presencia de la nutria y el $\mathrm{O}_{2}$ disuelto en el agua, aunque con bajo valor predictivo ya que la relación es directamente entre la abundancia de las especies presa y el $\mathrm{O}_{2}$ disuelto en el agua y la turbidez de la misma. El $\mathrm{O}_{2}$ disuelto disminuye por diversas causas, entre ellas la presencia de contaminantes en el agua o el aumento de sólidos suspendidos en ella (turbidez). Provocando que el metabolismo de los peces disminuya y en casos extremos llegando a la muerte, afectando así la abundancia de las especies-presa. Normalmente los parámetros de $\mathrm{O}_{2}$ en el agua para tener una población sana de peces es de $4-6$ $\mathrm{mg} / \mathrm{l}$ (Lloyd 1992). En el área de estudio el río Zimatán fue el que obtuvo la concentración más alta de $\mathrm{O}_{2}$ disuelto en el agua $(6.1 \pm 0.3 \mathrm{mg} / \mathrm{l})$.

La abundancia promedio para esta área entre los tres ríos con los modelos I y II fue de 0.39 nutrias $/ \mathrm{km}$, este valor es similar a los de otras áreas. Para México en Sonora se registra una abundancia de 0.34 nutrias $/ \mathrm{km}$ (Gallo 1996) para Argentina y Uruguay va de 0.81 a 2.76 nutrias/km (Parera 1996; Laeriviére 1999).

En este estudio se encontró que la abundancia de la nutria presentó variaciones dependiendo del modelo con el cual fueron analizados los datos. El modelo de Gallo (1996) ( I ) y el de Eberhardth \& Van Etten (1956) modificado ( II ) utilizan la tasa de defecación, lo que puede reducir el riesgo de sobreestimar la población, sin embargo, el modelo I no considera el tiempo de depósito, ya que fue hecho para llegar a la zona de muestreo, limpiarla y regresar al siguiente día al conteo de excretas, pero para lugares donde eso no se puede llevar a cabo se pierde el poder diferenciar a los grupos fecales nuevos y viejos, elevando el riesgo de sobreestimar la población. La tasa de defecación tiene una influencia muy importante en las estimaciones poblacionales de nutria, y ésta varía debido a diferentes factores, como la dieta, época del año, sexo y las condiciones en que se determinaron las tasas (cautiverio, número de animales y horas de observación). En Argentina se encontró que la tasa de defecación diaria es de 10 excrementos (Parera 1996b), mucho mayor a la reportada para México (Gallo 1996). Sin embargo al no haberse reportado una tasa de defecación para esta región de México o para los cuerpos de agua del bosque tropical caducifolio en México se tomó el único dato publicado para México y se combinó el resultado de los diferentes modelos para proporcionar una estimación más conservadora que cada modelo usado de forma independiente. 
Hábitos alimentarios. La nutria presenta una alimentación muy variada al igual que otras especies de los géneros Lontra y Lutra. Los resultados de este trabajo muestran una similitud con otros realizados en otras regiones (Adrian \& Moreno 1986; Clode \& Macdonal 1995; Kruuk et al. 1990; Ruiz-Olmos et al. 1989). En este estudio hubo cuatro grupos principales de presas (crustáceos, peces, anfibios e insectos) los cuales también se han reportado para otras zonas de América Latina. En Oaxaca se encontró una mayor depredación sobre los crustáceos que sobre otras presas, como en los trabajos realizados por Gallo (1989), Spínola (1994) y Parera (1996b) en Sierra Madre del Sur, México, Costa Rica y Argentina respectivamente. Esto porque los crustáceos son más abundantes en ambas épocas del año. Existen otros grupos que no se encontraron dentro de la alimentación de la nutria en esta región pero que han sido reportados en otros estudios, como las aves y los mamíferos (Spínola \& Vaughan 1995; Gallo 1989). Se encontraron algunas similitudes con los trabajos de Gallo (1989), Spínola \& Vaughan (1995) y Macías-Sánchez \& Aranda (1999), como la presencia de los crustáceos de los géneros Macrobrachium y Atya, los peces de la especie Agonostomus monticola, y de los géneros Awaous y Sicydium. Aunque no se encontraron aves ni mamíferos en los excrementos, sí se presentan en el área de estudio especies que podrían ser alimento de las nutrias, aunque probablemente no tengan la necesidad de depredar sobre estos organismos. En el caso de los anfibios por las características de las muestras quizá sean de la familia Hylidae (Zug 1993) la cual es abundante a la orilla de los ríos, con el mayor porcentaje de aparición en el río Ayuta durante la época de lluvia. Los excrementos en las que se encontraron restos de anfibios para la temporada seca corresponden a los primeros meses de ésta. Knudsen \& Hale (1968) mencionan que las nutrias a menudo comen insectos acuáticos y anfibios durante el invierno cuando la comida escasea, lo que seguramente ocurrió también con la ingesta de insectos para el río Ayuta pero con la sequía. Los insectos frecuentemente se encuentran en los excrementos aunque su contribución a la biomasa es pequeña. Normalmente las nutrias jóvenes se alimentan de insectos grandes como las ninfas de mosca y los escarabajos acuáticos, lo que coincide con lo encontrado en este estudio, en el que las ninfas de los coleópteros de la familia Corydalidae consumidos por las nutrias son grandes, variando en tamaño de 40 a $90 \mathrm{~mm}$ dependiendo de la especie (Borror et al. 1989). La presencia de insectos más pequeños es resultado de una ingesta secundaria o accidental (Melquinst \& Hornoker 1983). En este estudio la frecuencia de ocurrencia y la biomasa consumida no variaron mucho con referencia en las presas principales pero si en las presas con menor frecuencia de ocurrencia. Las principales presas de la nutria son nativas de México. M. americanum y M. acanthochirus pertenecen a la familia Palaemonidae, siendo los representantes del género Macrobrachium los que alcanzan las mayores tallas, en especial M. americanum que se puede pescar durante todo el año. En la época de lluvia se encuentra a lo largo de 
todo los ríos, durante la sequía se interrumpe el flujo de agua quedando zonas desprovistas de agua entre las partes altas del cauce, con flujo reducido pero constante y las partes bajas con ambientes de laguna costera. Su abundancia se incrementa durante la época seca, tiempo en el que habita las partes más altas donde encuentra las pozas más grandes y profundas (Gallo 1989). Este crustáceo es también muy consumido por la gente de la zona. Normalmente lo pescan a mano buscando en las hendiduras de las rocas del río, pero en raras ocasiones utilizan métodos poco selectivos, como es el echar cal en el agua (G. Cruz com. pers.).

Otra de las especies más consumidas por la nutria de río es el pez llamado "pega piedra" Gobiexos mexicanus, el cual pertenece a la familia Gobiesocidae. Habita en los ríos de las tierras bajas, donde tiene una distribución restringida y es escaso (Martínez-Ramírez 1999) se considera bajo protección especial de acuerdo a la NOM-059-SEMARNAT-2001.

A pesar de que la cuantificación de la abundancia relativa de las presas se realizó solamente durante mayo, julio y agosto, se capturaron gran parte de las especies existentes en el área. La relación entre la frecuencia de ocurrencia en los excrementos de las especies y la abundancia de éstas fue significativa, confirmando que la nutria es una especie de hábitos alimentarios generalistas, al aprovechar los recursos de acuerdo a su disponibilidad. La relación de la biomasa consumida de las presas y su abundancia fue de bajo valor predictivo, debido a que la relación se realizó con el porcentaje del volumen de los restos encontrados los cuales son valorados visualmente y no con el índice (frecuencia) que identifica por igual a las especies más importantes en la dieta.

Este estudio indica que por el tamaño poblacional aproximado de las nutrias en los ríos Ayuta, Copalita y Zimatán en la costa de Oaxaca son poblaciones viables (Soulé 1980) sin embargo, las presiones hacia las cuencas hidrológicas como la deforestación y la erosión consecuente pueden afectar la calidad del agua, lo mismo sucede con los fertilizantes y pesticidas de las prácticas agrícolas en incremento y el establecimiento de asentamientos humanos en las márgenes de los ríos, por lo que es un momento oportuno para establecer un sistema de monitoreo que permita conocer la fluctuación de la población de las nutrias y tomar medidas oportunas para su conservación. Es también necesario realizar estudios sobre las especies presa más consumidas ya que también son utilizadas por los habitantes del área por lo que se requiere que su explotación sea consistente con la conservación de las nutrias.

\section{AGRADECIMIENTOS}

Agradeciendo el apoyo financiero y logístico del Fondo Mundial para la Conservación de la Naturaleza (WWF). Sociedad para el Estudios de los Recursos Bióticos de Oaxaca (SERBO). Denver Zoological Foundation. Idea Wild, Inc. Laboratorio de Ictiología del 
Instituto de Biología UNAM. Al Dr. Brian Miller por todo su apoyo, a Cuauhtemoc Chávez por su ayuda en las cuestiones estadísticas, al Dr. Jorge Servín, Isaac Rojas y Emmanuelle Gamboa por su apoyo en el tema, así como a todos aquellos por lo que fue posible la realización de este trabajo. A la memoria de Leo Schibli.

\section{LITERATURA CITADA}

Adrian, M. I. \& S. Moreno. 1986. Notas sobre la alimentación de la nutria (Lutra lutra) en el embalse de Matavacas (Huelva). Doñana, Acta Vertebrata 13: 189-191.

Alho, C. J. R., T. E. Lacher, JR., \& H. C. Goncalves. 1988. Environmental degradation in the Pantanal ecosystem. Bioscience 38: 164-171.

Aranda, M. 2000. Huellas y rastros de los mamíferos grandes y medianos de México. Comisión Nacional para el Conocimiento y uso de la Biodiversidad. Instituto de Ecología A. C. Xalapa, Veracruz, México.

Arita, H. T. \& M. Aranda. 1987. Técnicas para el estudio y clasificación de los pelos. Instituto Nacional de Investigaciones sobre Recursos Bióticos. Xalapa, Veracruz, México.

Borror, D. L., C. A. Triplehorn, \& N. F. Jonson. 1989. An introduction to the study of insects. $6^{\mathrm{a}}$ ed. Philadelphia: Saunders Collage Publishing.

Carss, D. N. \& S. G. Parkinson. 1996. Errors associated with otter Lutra lutra faecal análisis. I. Assessing general diet from spraints. J. Zool. (London) 238: 301-317.

Casariego-Madorell, M. A. 2004. Abundancia relativa y hábitos alimetnarios de la nutria de río (Lontra longicaudis annectens) en la costa de Oaxaca, México. Tesis de Maestria. Posgrado en Ciencias Biológicas. Facultad de Ciencias. UNAM. México. 67 pp.

Ceballos G. \& A. García. 1995. Conserving neotropical biodiversity: the role of dry forests in western Mexico. Conservation Biology 9: 1349-1356.

Chehébar, C. 1991. Searching for the giant otter in northeastern Argentina. International Union for the Conservation of Nature, Otter Specialist Group Bull. 6: 17-18

Clode, D. \& D. W. Macdonal. 1995. Evidence for food competition between mink (Mustela vison) and otter (Lutra lutra) on Scottish islands. J. Zool. (London) 237: 435-444.

Contreras, F. 1994. Manual de Técnicas Hidrológicas. $1^{\text {a }}$ ed. Ed. Trillas. México D.F.

Eberhardt, L. L. \& R. C. Van Etten. 1956. Evaluation of the pelletgroup count as a deer census method. J. Wildl. Manage. 20:70-74.

Emmons, L. H. 1987. Comparative feeding ecology of felids in a neotropical rainforest. Behavioral Ecology and Sociobiology 20: 271- 283.

Franco-López, J. G. Aguero., A. Cruz., A. Rocha., N. Navarrete., G. Flores., E. Kato., S. Sánchez., L. Abarca., C. M. Bedia, e I. Winfield. 1985. Manual de Ecología. $1^{\mathrm{a}}$ ed. Ed. Trillas. México D.F.

Gallo, J. P. 1986. Otters in Mexico. J. of the Otter Trust 1: 19-24

. 1989. Distribución y estado actual de la nutria o perro de agua (Lutra longicaudis annectens Major, 1897) en la Sierra Madre del sur, México. Tesis de Maestría, Facultad de Ciencias UNAM.

. 1996. Distribution of the neotropical river otter (Lutra longicaudis annectens Major, 1897) in the rio Yaqui, Sonora, Mexico. IUCN. Otter Group Specialist Bull. 13: 27-31. 
García, G., S. Salas., L. Schibli., R. Aguilar., S. Acosta, \& A. Salazar. 1992. Análisis de la vegetación y usos actuales del suelo en el estado de Oaxaca. Fase I (Costa y Sierra sur).Informe técnico SERBO. AC.

Gori, M., G. M. Carpaneto \& P. Ottino. 2003. Spatial distribution and diet of the Neotropical otter Lontra longicaudis in the Ibera lake (northern Argentina) Acta Theriol. 48: 495-504.

Helder, J. \& K. De Andrade. 1997. Food and feeding habits of the neotropical river otter Lontra longicaudis (Carnivora, Mustelidae). Mamm. 61: 193-203.

INEGI. 1988. Carta de efectos climáticos. Juchitán. ED15-10D-15-1. Instituto Nacional de Estadística. Geografía e Informática.

Jacobsen, L. \& H. M. Hansen. 1996. Analysis of otter Lutra lutra spraints: Part 1: Comparison of methods to estimate prey proportions; Part 2: Estimation of the size of prey fish. J. Zool. (London) 238: 167-180.

Janzen, D. H. 1988. Tropical dry forest: the most endangered major tropical ecosystems. Pp. 130-137. In Biodiversity (E. O. Wilson, ed.). National Academy Press, Washington, D. C.

Knudsen, G. J. \& J. Hale. 1968. Food habits of otter in the Great lakes Region. J. Wildl. Manage. 32: 89-93.

Kruuk, H., D. Wansink, \& A. Moorhouse. 1990. Feeding patches and diving success of otters, Lutra lutra, in Shetland. Oikos 57: 68-72.

Laeriviére, S. 1999. Lontra longicaudis. Mamm. Species 609: 1-5.

Lloyd, R. 1992. Pollution and freshwater fish. A. Buckland Foundation Book. United States of America.

Macias-Sánchez, S. S. \& M. Aranda. 1999. Análisis de la alimentación de la nutria Lontra longicaudis (Mammalia: Carnivora) en el sector del río Los Pescados, Veracruz, México. Acta Zool. Mex. Nueva Serie 76: 49-57.

Martínez-Ramírez, E. 1999. Taxonomía y zoogeografía de la ictiofauna dulceacuícola del Estado de Oaxaca, México. Tesis de Doctorado. Universidad de Barcelona. Departamento de Ecología. Barcelona.

Melquist, W. E. \& M. G. Hornocker. 1983. Ecology of river otters in west central Idaho. $J$. Wild. Monographs 83: 1-60.

Montiel-Jaime, M. 1994. Biología y Ecología de los Gerreidos (Pisces: Gerreidae) de la laguna de Pueblo Viejo, Veracruz. Tesis de licenciatura. FES. Zaragoza. UNAM. México. D. F.

Myers, R. H. 1986. Classical and Modern Regression with Applications. Duxbury Press. Boston, Massachusetts.

Pardini, R. \& E. Trajano. 1999. Use of shelters by the neotropical river otter (Lontra longicaudis) in an Atlantic forest stream, southeastern Brazil. J. Mamm. 80: 600-610.

Parera, A. 1996a. Estimating river otter Lutra longicaudis population in Iberá lagoon using a direct sightings methodology. IUCN Otter Specialist Group Bull. 13: 77-83.

— 1996b. Las nutrias verdaderas de la Argentina. Fundación Vida Silvestre Argentina. Boletín técnico 21: 13-20.

Pascual-Toca, M. 2000. Variaciones estacionales en la dieta de la nutria (Lutra lutra) en la cuenca del río ESVA (Asturias). Seminario de Investigación dentro del Programa de Doctorado Organismos y Sistemas Forestales y Acuáticos del Departamento de Biología de Organismos y Sistemas de la Universidad de Oviedo. 
Ruiz-Olmos, J., G. Jordán, \& J. Gonsalbez. 1989. Alimentación de la nutria (Lutra lutra L. 1758) en el Nordeste de la Península Ibérica. Doñana Acta Vertebrata 16: 227-237.

Ruiz-Olmos, J., D. Saavedra, \& J. Jiménez. 2000. Testing the surveys and visual and track censuses of Eurasian otters (Lutra lutra). Zoological J. of the linnean Society of London 253: 359-369.

Ruiz-Olmos, J., J. Jiménez., S. Palazón., M. Delibes., C. Bravo, \& F. Bueno. 1998. Factores que han determinado la situación actual de las poblaciones de nutria y propuestas de gestión. Pp. 223-242. In La nutria en España ante el horizonte 2000 (J. Ruiz-Olmo, y M. Delibes, eds.). Sociedad Española para la Conservación y Estudio de los Mamíferos (SECEM) Grupo Nutria. Barcelona-Sevilla-Malaga.

Rzedowski, J. 1978. Vegetación de México. Ed. Limusa, México.

Salas-Morales, S. H. 2002. Relación entre la heterogeneidad ambiental y la variabilidad estructural de las selvas tropicales secas de la costa de Oaxaca, México. Tesis de Maestira. Facultad de Ciencias, UNAM. D.F.

Salas, S., E. Torres., A. González., L. Schibli., H. Morales, \& M. Cerón. 1996. Análisis de la vegetación y uso actual del suelo en el estado de Oaxaca, Fase IV (Istmo). Informe Técnico. SERBO. A. C. Oaxaca (No publicado).

SEMARNAT 2002. Norma Oficial Mexicana NOM-059-ECOL-2001. Protección ambiental - especies nativas de México de flora y fauna silvestres - Categorías de riesgo y especificaciones para su inclusión, exclusión o cambio - Listas de especies en riesgo. Diario Oficial, 6 de marzo de 2002, 56 pp.

Sokal, R. \& J. Rohlf. 1981. Biometry. $2^{\text {a }}$ ed. W. H. Freeman y Co. San Francisco.

Soulé, M. E. 1980. Thresholds for survival: Maintaning fitness and evolutionary potential. Pp. 151 - 169. En M. E. Soulé y B. A. Wilcox (eds.). Conservation Biology; An Evolutionary-Ecological Perspective. Sinauer Associates, Sunderland, Massachussets.

Spinola, R. 1994. Dieta, abundancia relativa y actividad de marcaje de la nutria neotropical (Lutra longicaudis) en la Estación Biológica La Selva, Costa Rica. Tesis de Maestría. Programa Regional en Manejo de Vida Silvestre para Mesoamerica y el Caribe, Universidad Nacional. Heredia, Costa Rica.

Spinola, R. \& C. Vaughan. 1995. Dieta de la nutria neotropical (Lutra longicaudis) en la estación biológica la Selva, Costa Rica. Vida Silvestre Neotropical 4: 125-132.

Texeira, R. L. 1994. Abundance, reproductive period, and feeding habits of eleotrid fishes in estuarine habitats of north - east Brazil. J. of fish Biol. 45: 749-761.

Toweill, D. E \& J. E. Tabor. 1982. River otter: Lutra canadensis. Pg. 688?703 In J. A. Chapman and G. A. Feldhamer (eds). Wild mammals of North America: biology, management, and economics. Johns Hopkins University Press. Baltimore, MD.

Wise, M. H. 1980. The use of fish vertebrae in scats for estimating prey size of otters and mink. J. Zool. (London) 192: 25-31.

Zar, J. H. 1999. Biostatistical Analysis. Prentice-Hall, Upper Saddle River, NJ.

Zug, G. R. 1993. Herpetology and Introductory biology of amphibians and reptiles. 4a ed. Academic Press. INC. San Diego California. 
